Aus Sanitätsrat Dr. Weickers Volkssanatorium ,Krankenheim".

\title{
Striae distensae in der Haut des Rückens bei Lungenkranken.
}

\author{
Von
}

Dr. Warnecke, Görbersdorf.

Mit 1 Tafel.

Das Auftreten von Striae distensae oder atrophicae in der Haut des Rückens bei Lungenkranken ist scheinbar bisher wenig beachtet.

Ich wurde zuerst 1909 durch meinen verstorbenen Lehrer Dr. L. von Muralt auf die Striae aufmerksam gemacht. Seitdem habe ich diese Hautveränderung noch einigemal beobachten können. Da die Ätiologie der Striae bei Lungenkranken unklar ist und sich in der Literatur nur sehr wenig darüber findet, möchte ich über diese interessante, immerhin seltene Erscheinung berichten.

Die Striae als solche sind ein alltägliches Vorkommnis. R i ecke (1) schreibt darüber folgendes :

„Im Verlnuf der Gravidität, sowie bei Personen mit rascher Zunahme des Fettpolsters, können namentlich am Abdomen, aber auch an den Oberschenkeln und der Schultern streifenförmige Veränderungen der Haut auftreten, die mehr oder weniger scharf begrenzt sind und eine glatte, leicht eingesunkene Oberfläche von weisser oder blaulich-weisser Farbe haben. Ihre Verteilung ist symmetrisch, ihre Anordnung im einzelnen eine parallele. Diese Striae kommen durch eine Ưberdehnang der Haut zustande, der das elastische Gewebe, wenn es nicht völlig intakt ist, nicht $z u$ folgen vermag. Am Rande der Herde findet man die geborstenen elastiscben Fasern aufgerollt. Die beschriebenen Erscheinungen kommen meist bei jugendlichen Individuen und haufiger beim weiblichen Geschlecht vor."

Das Auftreten der Striae bei schnell wachsendem Aszites und bei Tumoren ist gleichfalls bekannt.

Auf die histologischen Vorgänge bei Entstehung der Striae will ich nicht näher eingehen. Die Untersuchungen von Passarge (2), Langer (3), Unna (4), Zieler (5) und von anderen Autoren geben darüber Aufschluss.

Die Literatur über die Striae bei Lungenkranken ist, soweit sie mir zur Verfügung stand, sehr.spärljcb. Öfter 
beschrieben ist das Vorkommen der Striae als Begleiterscheinung bei 'Typhus abdominalis (Striae patellares oder praepatellares) und bei anderen langdauernden fieberhaften Erkrankungen.

Des allgemeinen Interesses wegen möchte ich kurz darauf oingehen.

Kirstein (6) erwähnt, dass bereits 1835 Ascherson (7) in Berlin und nach thm in den Jahren 1856-63 Reuss (8), Röser (9) und Förster (10) insgesamt 13 Fälle dieser merkwürdigen Hautaffektion beobachtet haben. Meist handeite es sich um Striae an den Extremitäten bei I'yphus, Typhlitis, Dysenterie etc. 1893 stellte $\mathrm{Kirstein}$ in der medizinischen Gesellschaft in Berlin einen $18 \mathrm{jähr}$. jungen Mann yor mit querverlaufenden Striae auf dem Rücken links von der Höhe des 10 . Brustwirbels bis zur oberen Grenze des Darmbeinkammes. Die Striae waren nach einer Perityphlitis während einer 7 wöchigen Bettruhe entstanden, und da der Kranke gleichzeitig eine doppelseitige tuberkulöse Lungenaffektion hatte, nahm Kirstein ein tuberkulöses Darmleiden an. Während Kirstein in der Sitzung den Fall als T'rophoneurose vorstellte, spricht er in der Veröffentlichung nur von einer verminderten Widerstandsfähigkeit und einer mechanischen Dehnung der Haut.

$F$ is cher (11) beschuldigt als ätiologische Momente verschiedene Dinge: rasches Knochenwachstum, infolge einer bestehenden Neuritis trophisch schlecht innervierte Hautlecken, das Hinaufziehen der Knie und dadurch bedingte Anspannung der Haut.

Köbner (12) beobachtete ebenfalls die Striae distensae bei Typhus und hält besonders auf Grund seiner histologischen Untersuchungen die mechanische Dehnung für die einzige in Betracht kommende Ätiologie. In seiner Arbeit findet sich die Notiz, dass 1867 Dr. A uerbach ihm persönlich über Striae am Thorax eines 15jährigen, an Tuberkulose erkrankten jungen Mannes berichtet habe. Es dürfte dies der älteste Fall von Striae distensae in der Rückenhaut eines Lungenkranken sein, der in der Literatur Erwähnung findet.

Sieveking (13) sah an den Knien die Striae je einmal bei Typhus und bei Meningitis, Reiche (14) bei. Meningitis cerebrospinalis, von Hansemann (15), Strasser (16) und Kaiser (17) beobachteten die Striae patellares bei Abdominaltyphus: Ka iser fand unter 1932 Typhusfüllen 25 Kranke mit den Striae an der Kniescheibe. Er sah dieselben auch bei einem Fall von Ruhr und von Lungentuberkulose. Immer handelte es sich um jugendliche Personen:

$\mathrm{Ka}$ is er nimmt für die Entstehung der Striae das rein mechanische Moment an, besonders verschiedene Wachstumsverhältnisse zwischen Knochen und Haut der Oberschenkel.

Ähnliche Vorstellungen haben Schottmïller (18) und Feer (19).

Jessner (20) erwihnt das Vorkommen der Striae dieser sehr $^{\text {sem }}$ seltenen Erscheinung " bei Lungenentzündung, und zwar weiss or nur einen Fall anzuführen (The Practitionner, Sept. 1908). „Am Tage nach der regulären Krisis zeigten sich zu beiden Seiten der Wirbelsäule quer über den Rücken laufende narbenähnliche Streifen, die 
ungefähr dem Verlauf der Rami dorsales der dem erkrankten Gebiete zugehörigen Thorakal- resp. Lumbalnerven entsprachen." Fieber".

Bouchard (21) beobachtete Striae im Anschluss an "typhöses

Sattler (22) zeigte 1910 in einer Sitzung der Gesellschaft für innere Medizin und Kinderheilkunde in Wien ein 14 jähriges Mädchen; bei dem im Gefolge eines zweimal rezidivierenden 'Typhus längsverlaufende Striae an den Vorder- und Innenflächen der Oberschenkel und am Gesäss"aulgetreten waren. Sattler nimmt an, dass eine trophoneurotische Schädigung der Haut und starkes Längenwachstum der langen Röhrenknochen eine Rolle spielten. Gleichzeitio fanden sich bei der Kranken trophoneurotische Störungen an den Fingernägeln (Riefung), blave Verfärbung und Hyperbidrosis der Hände.

Matthes (23) bat die Striae praepatellares ebenfalls bei einem Fall von Typhus abdominalis gesehen. Er erwähnt zur Erklärung nur die Möglichkeit, dass die Knie längere Zeit während des Fiebers gebeugt gehalten und dadurch die Haut der Streckseite gespannt wurde.

Bleibtreu (24) beschreibt bei, einem 15 Jahre alten Mädchen mit Scharlach symmetrische quergestellte Striae auf dem Gesäss und an den Knien. Bleibtreu möchte mechanische und trophoneurotische Einflüsse ablehnen.

Von E. Freund (25) wurde 1913 in der Wiener medizinischen Gesellschaft ein Mann vorgestellt mit Striae distensae auf dem Rücken nach Sepsis, die sich im Anschluss an eine Angina entwickelt hatte (prälaryngeale Abszesse, Pneumonie, Milztumor, Gonitis, Polyneuritis). Es fanden sich bis $10 \mathrm{~cm}$ lange und $1 \mathrm{~cm}$ breite atrophische Hautstreifen, in deren Bereich die Sensibilität herabgesetzt war. Freund führt die Streifen darauf zurück, dass der Kranke lange bettlägerig war.

Von französischen Autoren sind im ganzen 7 Fälle von Striae bei Lungenkranken veröffentlicht und von Cramer (26) 1913 zusammengestellt worden. Die folgende Tabelle mag darüber orientieren:

\begin{tabular}{|c|c|c|c|}
\hline Cramer & $\begin{array}{l}17 \mathrm{~J}, \\
\mathrm{~m} .\end{array}$ & $\begin{array}{c}\text { r. Spitze. r. Plenritis. Mitralstenose. Thorax- } \\
\text { hälfte r. }<1 .\end{array}$ & Striae links \\
\hline Thaon & $\begin{array}{l}17 \mathrm{~J} ., \\
\mathrm{m} .\end{array}$ & $\begin{array}{l}\text { r. käsige Pneumonie pnd natürlicher Pneumo- } \\
\text { thorax. Striae die Wirbelsäule überschreitend. }\end{array}$ & $\pi$ \\
\hline Trossat & $\begin{array}{l}15 \mathrm{~J} ., \\
\text { w. }\end{array}$ & $\begin{array}{l}\text { r. tuberkulöse Pleuritis. Striae an den Ober- } \\
\text { armen, am Bauch und an den Oberschenkeln, } \\
\text { etwa } 1 \text { Monat nach der Pleuritis aufgetreten. } \\
\text { Thoraxhälite } r .<1 \text {. }\end{array}$ & $\pi$ \\
\hline Gimbert & $\begin{array}{l}17 \mathrm{~J} . \text {, } \\
\mathrm{m} .\end{array}$ & $\begin{array}{l}\text { Tuberkulose der r. Lunge und Pneumothorax. } \\
\text { l: Spitze. Thoraxhälfte r. }<1 .\end{array}$ & $*$ \\
\hline Gilbert & $\begin{array}{l}17 \mathrm{~J} ., \\
\mathrm{m} .\end{array}$ & $\begin{array}{l}\text { 1. Pneumonie und Pleuritis. Typhus abdomi- } \\
\text { nalis. Thoraxbälfte r.< l. Striae neben der } \\
\text { Kniescheibe. }\end{array}$ & Striae rechts \\
\hline Troisier & m. & $\begin{array}{l}\text { Lungentuberkulose r. }<\text { l. 1. Kaverne und } \\
\text { Adhäsionen. Striae am Oberschenkel. }\end{array}$ & $n$ \\
\hline Seialom & $\begin{array}{l}20 \mathrm{~J} ., \\
\mathrm{m} .\end{array}$ & $\begin{array}{l}\text { r. Tuberculosis pulmonum. Striae die Wirbel- } \\
\text { säule überschreitend. Thoraxhälfte gleich. }\end{array}$ & Striae links \\
\hline
\end{tabular}


Die französischen Autoren nehmen eine mechanische Ursache, eine übermässige Dehnung der Haut an, zum Teil in Verbindung mit einer trophischen Störung (Thaon, Trossat). Cramer möchte lediglich die Distension der Haut ätiologisch verantwortlich machen. Er fand die Striae in don von ihm zusammengestellten Fällen immer auf der der pulmonalen und pleuralen Luision entgegengesetzten Seite. Sensibilitätsstörungen wurden niemals nachgewiesen, dagegen bei den 7 Fällen viermal eine messbare Differenz der Thoraxhälften. $\mathrm{Cr}$ amer vermutet, dass die kompensatorische Erweiterung der gesunden Lunge und die daran anschliessende Ausdehnung der Brusthïlfte die Haut ühermässig gedehnt hat, und dass so die Striae rein mechanisch zustande gekommen sind. Auch langes Liegen auf der kranken Seite führt seiner Ansicht nach zu einer kompensatorischen Dilatation der gesunden Thoraxhälfte.

Ich habe im Laufe der Jahre an dem Material der Dr. Weickerschen Volksheilstäte 11 Fälle von Striae des Ruckens und einen Fall ron Striae an den Schultern boobachten können.

\begin{tabular}{|c|c|c|}
\hline 1. B., $\begin{array}{c}49 \mathrm{~J} ., \\
\mathrm{m} .\end{array}$ & $\begin{array}{c}\text { Doppelseitige Erkrunkung der Spitzen. Tbe. - . Vor } \\
26 \text { J. erkrankt. Neurasthenie. }\end{array}$ & Strine rechts \\
\hline 2. $\left|\begin{array}{c}\text { M.. } 25 \mathrm{~J} ., \\
\mathrm{m} .\end{array}\right|$ & $\begin{array}{l}\text { Doppelseitige Erktankung der Spitzen. 'I'bc. - } \\
\text { Chron. Bronchitis. Ausgedehnte Drissentuberkulose. }\end{array}$ & $n$ \\
\hline $\begin{array}{c}\text { 3. Scli, } 24 \text { J., } \\
\text { in. }\end{array}$ & $\begin{array}{l}\text { Rechte Spitze leicht, linke Iunge nusgedehnt erkrankt. } \\
\text { Starke Schrumpfung der linken I'horaxhilfte. I'be. }+ \text {. } \\
\text { Ca. 2 J. krank (Striae em Bnuch, Oberschenkeln, } \\
\text { Oberarmen, Geshss). }\end{array}$ & Striae links \\
\hline 4. K., $23 \mathrm{~J} .$, & $\begin{array}{l}\text { Bds. schwere Lungenerkrankung. Tbc. +. Naben- } \\
\text { hoden-Tuberkulose. } 2 \mathrm{~J} \text {. krank (v gl. Abbild. 1). }\end{array}$ & Strine rechits \\
\hline 5. K.. $18 \mathrm{~J}$. & $\begin{array}{l}\text { Bds. Oberlappen-Erkrankung, links vorgeschrittene } \\
\text { exsudative Prozesse, links unten Randgeriusche. } \\
\text { Tbc. +. Öfter Lungenblutungen. } 1 \mathrm{~J} \text {. krank. }\end{array}$ & Strige Jinks \\
\hline $\begin{array}{l}\text { 6. } 0 ., 26 \mathrm{~J} ., \\
\mathrm{m} .\end{array}$ & $\begin{array}{l}\text { Dappelseitige schwere Erkranknng, rechts pleuritische } \\
\text { Schwarte, links Unterlappen-Urozess. T'bc. +. Seit } 1 \mathrm{~J} \text {. } \\
\text { krank. Blutungen. Vor } 1 \text { J, rechts Pleuritis exsudativa. }\end{array}$ & Striae rechts \\
\hline$\underset{\mathrm{m} .}{\mathrm{W} ., 18 \mathrm{~J} .}$ & $\begin{array}{l}\text { Rechts alte Oberlappen-Erkrankung, links nusge- } \\
\text { dehnte frische Erkrankung der ganzen Lange. Tbc. +. } \\
\text { Starke Blutungen. Fieber. Vor } 2 \mathrm{~J} \text {. links Pleuritis } \\
\text { exsudativa. }\end{array}$ & Striae links \\
\hline 8. $\frac{\text { Sch., } 20 \mathrm{~J} .,}{\mathrm{m} .}$ & $\begin{array}{l}\text { Bds. schwore Erkrankung r. }<1 \text {. Links Ripponfell- } \\
\text { entzundung. Tbc. +. Erkrankt vor } 1 \% \text { J. Die } \\
\text { Striae sind vor fast } 1 \text { J. ontstanden, sio liberschreiton } \\
\text { die Wirbetstiule. Geringe Differenz dor Thoraxhalfte. } \\
\text { r. }>1 .\end{array}$ & Strise recits \\
\hline $\begin{array}{l}\text { 9. J., } 18 \mathrm{~J} ., \\
\mathrm{m} .\end{array}$ & 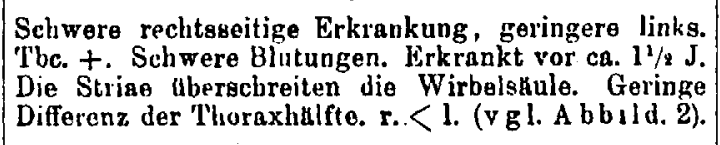 & Strine links \\
\hline $\begin{array}{l}\text { 10. } \\
\text { N., } 26 \mathrm{~J} ., \\
\mathrm{m} .\end{array}$ & $\begin{array}{l}\text { Rechts geringe, links ausgedelınte Erkrankung. Tbc. }+ \text {. } \\
\text { Vor ca. } 6 \text { J. erkrunkt. Blutungen. Die Striae Hber- } \\
\text { schreiten die Wirvelskule, reichin etwa vom } 10 \text {. Brust- } \\
\text { bis } 3 \text {. Lenden-Wirbel. }\end{array}$ & $n$ \\
\hline
\end{tabular}




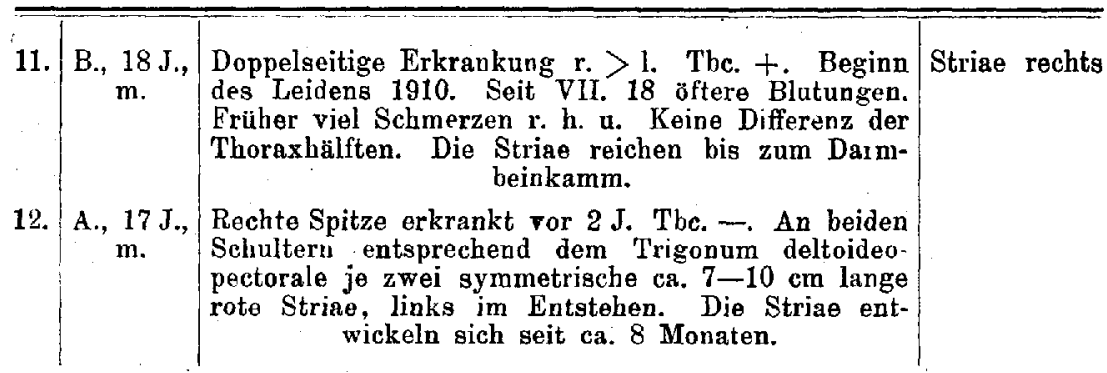

Die Durchsicht der in kurzem Auszug mitgeteilten Krankengeschichten ergibt ohne weiteres, dass wir jede Überdehnung der Haut beider Entstehung der Striae ausschliessen können. Es bestanden zwar in Fall 8 u. 9 messbare Differenzen im Umfang der Thoraxhälften und die Striae fanden sich auf der gesunderen dilatierten Seite, aber gerade bei diesen beiden Kranken überschritten die Striae die Wirbelsäule. Im Fall 3 war ebenfalls eine erhebliche Schrumpfung der linken Seite infolge einer Pleuritis exsudativa nachzuweisen, aber die Striae waren über der Pleuraschwarte aufgetreten. In den Fällen 3, 5, 6, 7, 10, 11 sah man die Striae auf der kränkeren Seite, dreimal über einer abgelaufenen exsudativen Pleuritis.

Die Striae verliefen in 2 bis zu 11 mehr oder weniger zusammenhängenden Reihen untereinander angeordnet, parallel zu den Rippen. Sie gingen von der Wirbelsäule bis zur hinteren und mittleren Axillarlinie und reichten in 2 Fällen über die. Wirbelsäule hinaus auf die andere Rückenhälfte.

Sichere Angaben über die Zeit der Entstehung der Striae konnten die Kranken nicht machen. Sie wussten zum T'eil nichts von dem Vorhandensein derselben. Nach der Farbe der Narbenzüge zu urteilen - von rot-violett bis blass-bläulich oder weiss - handelte es sich im Fall 1 wahrscheinlich um ganz alte, bei allen anderen Kranken um frischere Vorgänge. Fall 8 konnte angeben, dass die Striae vor einem Jahr, etwa 3 Monate nach Beginn seines Leidens zuerst beobachtet wurden. Bei dem 17 Jahre alten jungen Mann (12) waren die Striae vor 8 Monaten aufgetreten. Dieser Fall erscheint durch den symmetrischen Sitz der Striae nicht auf dem Rücken, sondern vorn über dem Trigonum deltoideo-pectorale besonders bemerkenswert. Auf der linken Seite waren die blauroten Striae offenbar noch in der Entwickelung begriffen. Gewichtszunahmen waren hier nicht zu verzeichnen. Im Fall 10 u. 11 reichten die ziemlich blassen Striae beiderseits tief herunter bis zur Lendengegend.

Auffallendere Zunahmen des Fettpolsters waren in den Anamnesen nicht angeführt, nur Fall 3 hatte ein höheres Körpergewicht $(74 \mathrm{~kg}$ und $20 \mathrm{~kg}$ Gewichtsabnahme). Wenn auch bei diesem Kranken die Striae an den Extremitäten und dem Bauch auf schnelles Anwachsen des Fettgewebes zurückgeführt werden könnten, so doch sicher nicht die auf dem Rücken vorhandenen.

Bemerkenswert ist es, dass die Striae fast immer nur bei jugend- 
lichen Personen, und zwar ausschliesslich bei männlichen Kranken gefunden wurden.

Zum Teil waren die Kranken lüngere Zeit bettligerig gewesen. Eine besondere Lage hatten sie im Bett nachweislich nicht bevorzugt.

Sensibilitätsstörungen waren in keinem Fall nachzuweisen, dagegen bestanden zum Teil gewisse Zeichen einer „nervösen reizbaren Schwäche".

Wenn - wie schon erwähnt - für unsere Fälle das mechanische Moment für die Entstehung der Striae nicht herangezogen werden kann, so fragt sich, ob wir irgend eine andere $\ddot{\Lambda}$ tiologie für diese auffallende. Erscheinung verantwortlich machen können. Für die Seltenheit des Vorkommens spricht der Umstand, dass den 12 Fällen ein Krankenmaterial von etwa 2000 tuberkulösen Männern zugrunde liegt, und dass bei etwa 4000 kranken Frauen und Mädchen die Striae in der Rückenhaut überhaupt nicht beobachtet wurden.

Es wurde bereits angeführt, dass bei Infektionskrankheiten, bei 'lyphus abdominalis, bei Typhlitis, bei Dysenterie, bei Sepsis, bei Pneumonie, bei Meningitis und bei Skarlatina Striae distensae beschrieben sind, für die man bisher'zum 'Teil keine rechte Frkliirung gefunden hat. Endlich wurde von Cantani (27) einmal, von Féré und Quermonne (28) in 2 Fällen das Auftreten von Striae am Körper von Neuropathen gesehen, und zwar an den Extremitäten, der Lendengegend, am Bauch, am Schulterblattwinkel und an den Brüsten. Auch diese Autoren lehnen den traumatischen Ursprung der Striae ab und nelimen an, dass bei iliren. nenropathischen Kranken ein Mangel an Widerstandsfïhigkeit der Haut vorgelegen hat. "S'agit-il lì d'un trouble trophique en rapport avec l'itat du système nerveux ou d'une défant de résistance congénitale de la peau coincident."

Man wird nicht über Vermutungen hinauskommen, wenn man die Striae bei Lungenkranken auf neurogene Vorgänge zurückführen will.

Eine Teilfunktion des vegetativen Nervensystems ist die trophische, mag man die trophische Alleinfunktion einzelner Nerven anerkennen oder nicht. Die Trophik des Nervensystems reguliert den Zellstofíwechsel und beeinflusst die Lebensfäbigkeit und die Widerstandskraft der Gewebe und der Gewebsbestandteile gegen äussere Schädigungen usw. Die trophische Nerrenfunktion scheint in hohem Masse von dem Apparat der Blutdrüsen abhängig zll sein. Sie wird wie alle übrigen Nervenfunktionen einer konstitutionellen Beeinflussung, einer reizbaren Scliwächo unterliegen können. Erkrankungen und Anomalien der Hant sind zum grossen 'T'eil Ausdruck von Störungen und Dystrophien innerer Organe. Es gibt zweifellos eine generelle zu Hauterkrankungen disponierende Konstitutionsanomalie, eine minderwertige Veranlagung des gesamten Integuments mit allen seinen einzelnen T'eilen. Die konstitutionellen Varietäten der Hautbeschaffenheit werden in weitestem Masse mechanischen, neurotrophischen, endokrinen und toxischen Einflüssen zugänglich sein. Das Hautorgan 
bietet der Erforschung konstitutioneller Differenzen ein weites Feld. Ich verweise auf die Zusammenstellung in B auers (29) vortrefflichem Lehrbuch.

Bei der Lungentuberkulose sind Hautveränderungen - ich meine hier nicht die tuberkulösen Hauterkrankungen - durchaus nicht selten. Meist handelt es sich um Anomalien auf toxischer Basis, um vasomotorische, von der inneren Sekretion abhängige Störungen; daneben sind bekannt allgemein neurotrophische Veränderungen. Ich erinnere an die immerhin selteneren trophischen Störungen im Bereich des Halssympathikus, an die Pigmentverschiebungen im Gesicht von Lungenkranken (Chloasma), an die Hemiatrophia faciei (Jacquet), an die stärkere Pigmentierung der Iris auf der kränkeren Seite [Herbert (30)]. Es gehören hierher der von Frédéricq und Thompson (31) beschriebene rote Zahnfleischsaum, die Neigung zu Zahnkaries, die Anomalien der Nägel. Die bläuliche Verfärbung der Nägel der Lungenschwindsüchtigen war schon von Hippokrates (unguis Hippokratis) beobachtet. Der Haaransfall, das frühzeitige Ergrauen, die Trockenheit und Glanzlosigkeit der Haare, die Frostbeulen [vgl. v. Muralt (32)], alles dies sind Erscheinungen, die wir zu den Störungen der trophischen Nervenfunktion zu rechnen haben. Die Beziehungen des Herpes zoster, den wir gar nicht so selten bei Lungenkranken antreffen, zu abnormen Zuständen des Nervensystems, des Spinalganglions oder des peripheren Nerven sind hier anzuführen.

Es liegt in der Natur der Sache, dass die Untersuchung der trophischen Funktion der Nerven und die Feststellung vieler neurotrophischer Störungen auf grosse Schwierigkeiten stossen müssen. Die den beschriebenen Striae zugrunde liegende Schädigung müsste im. Bereich der Thorakal und Lumbalnerven oder den zugehörigen Intervertebralganglien zu suchen sein. Die krankhaften Vorgänge würden event. toxisch entzündlich sein oder mechanisch durch Zerrung, Verwachsungen, Schwielenbildung etc. bedingt werden. Mit Sicherheit dürfen wir erwarten, dass einmal konstitutionelle Schwankungen der trophischen Nervenfunktion, andererseits eine angeborene Schwäche und Minderwertigkeit des betreffenden Erfolgsorgans eine grosse Rolle spielen müssen. Unsere Erfahrungen auf diesem weiten Gebiet sind noch sehr dïrtig, wenn auch gerade die Erforschung der krankhaften Veränderungen der vasomotorisch-trophischen Nervenfunktion reich an neuer Erkenntnis gewesen ist.

So sind wir vielleicht berechtigt bei der Entstehung der Striae in der Rückenhaut der Lungenkranken an eine neurogene Ursache zu denken, wenn wir auch den endgültigen Beweis dafür einstweilen schuldig bleiben müssen.

Nur umfangreichere Beobachtungen mit Sektionsmaterial könnten völlige Klarheit schaffen.

Noch einmal möchte ich zum Schluss erwähnen, dass die meisten Autoren, so besonders auch Zieler auf Grund seiner pathologischanatomischen Untersuchungen speziell für die Striae distensae bei Typhus als hauptsächlichste Ursache die mechanische Dehnung der Hant ansehen und die Annahme einer Trophoneurose ablehnen. Dem- 
gegenüber erscheint es mir unmöglich, dass für die Striae der Rückenhaut bei Lungenkranken die Dehnung der Haut allein verantwortlich gemacht werden könnte.

\section{Literatur.}

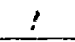

1. Riecke, Lehrbuch der Haut- und Geschlechtekrankheiten. Jena 1909. S. 339.

2. Pasaarge und Krbsing, Schwnd und Regeneration des elast. Gewebea der Hant unter versch. pathol. Verhitltnissen. Dermat. Studie. Haunlsurg. Leipzig. Voss. 1894.

3. Langar. Med. Jahrbuch 1880 . S. 49-57.

4. Un na, Histopathologio der Hautkrankheiten 1894. S. 1011-15.

5. Zieler. Zur l'athogenese der Dohuungsstroifen der Haut. (Strine cutis distense.) Manch. meil. Wochenschr. 1905. 37. S. 1764.

6. Kirstein, Uber streifenformige Divulsion der Hnut als Bcgleiterscheinung schwerer fieberhafter Kıaukheiten. Berl. klin. Wochenschr. 1s93. 41. S. 988.

7. Ascherson, 8. Rausa, 9. Rüser, 10. Furater. zit. Kirstein, 1. c

1i. Fischer, Striae patellares nach Typhus. Munch. med. Wochenselur. 1904. 11. S. 482

12. Kö bner, Ober Striae cutis distensae. Manch. med. Wochenschr. 1904. 21. s. 928.

13. Siereking, Jahrb. d. Hamb. Staatskrankenaust. III. S. 381.

14. Reiche, ZentralbJ. f. klin. Med. 1893.

15. v. Hansemann, Nach schriftl. Mitteilung.

16. Strasser. Ein bemerkenswerter F́all von Striat distensae cat. Mod. Klinik 1916. 2 i. S. 722 .

17. Ka iser. Strias patellares nach Bauchtyphus. Med. Klinik 1916. 46. S. 1206.

18. Sch ot tmillor: Mohr und Stähelin, Handb. d. inn. Mod. 1911. Bd. 1. S. 394. 417.

19. Feer, Lehrh. d. Kinderkrankbeiten. Jema. Fischer 1911, S. 610.

20. Jesgner, Dermat. Vortr. H, 22. Wurzburg, Kabitzech 1911. Hautverianderungen bei Erkrank. der Atmungeorgane.

21. Bouchard. Des verget. au niveau des articul. surven, au déclin de la fièrre typhoïde chez les adoleac. Bull. de In Soc. clin. 1879.

22. Sattler, Ref. Munch. med. Wochenschr. 1910. 21. S. 1157.

23. Mat thes, Lehrb. d. Diff. Diagn. inn. Krankb. Berlin 1919. S. 22.

24. Bleibtrou, Zwei seltene Beobachtungon bei Scharlach. Munch mod. Woohenschr. 1905. 37. S. 1767.

25. E. Freund, Ref. Munch. med. Wochenechr. 1914. 1. S. j1.

26. Cramer. Les vergetures du thorax. Revue de Médecin 1913. 23. S. 508-14 (dort Literatur) ref. Internat. Zentrulbl, f. d. ges. 'I'bc.-Forschung 1915. \$. 236.

27. Cautani, Zit. Eóré et Quermonne.

28. Ch. Féré et $J_{\text {. }}$ Ou ormonde, Notes sur vergetures do la peau recontróes chez des névropathes. le progròs médical. 1881. 44. S. 837.

29. Bauer, Die konstit. Disposition zu inn. Krankheiten. Berlin 1917.

30. Jacquet, Herbert, Zit. Jessen. Lungenschwindsucht und Nervensystem. Fischer, 1905.

31. Frédéricq u. Thompson, Zit. Turban. Beitr. z. Kenntnis d. Lungentbe. Wiesbaden 1899 .

32. L. v. Muralt, Die nervösen und psych. Störangen der Lungentuberkulosen. Med. Klinik 1913. H. 44 u. 46. 


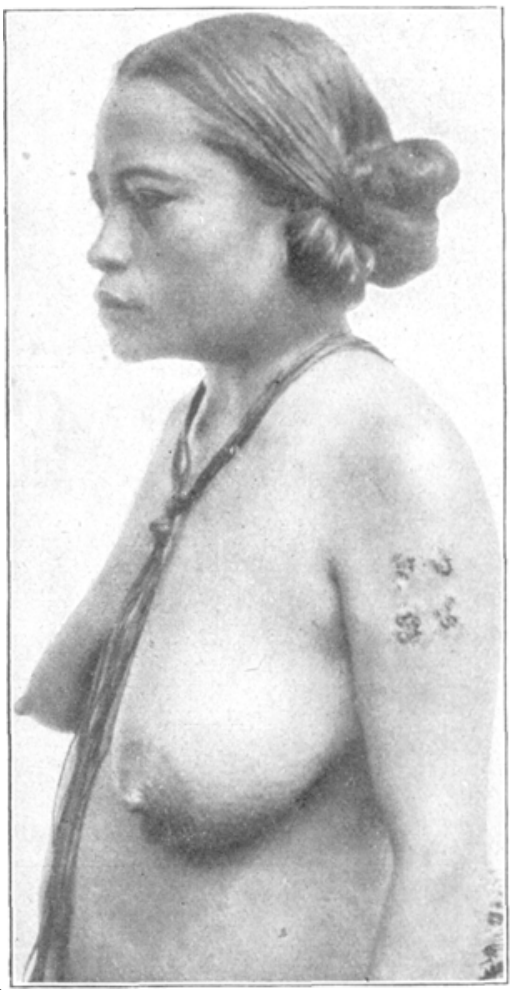

Fig. 1. Karolinerin am 6. Tage nach der Pirquetimpfung.

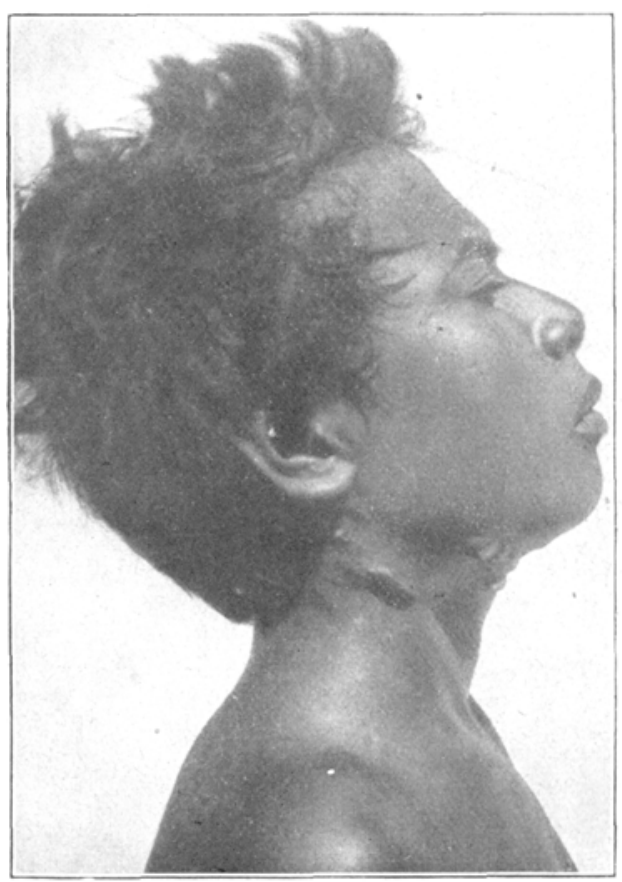

Fig. 3. Knabe mit fistuloser Kiefer- und Halsdrüsenskrofulose.

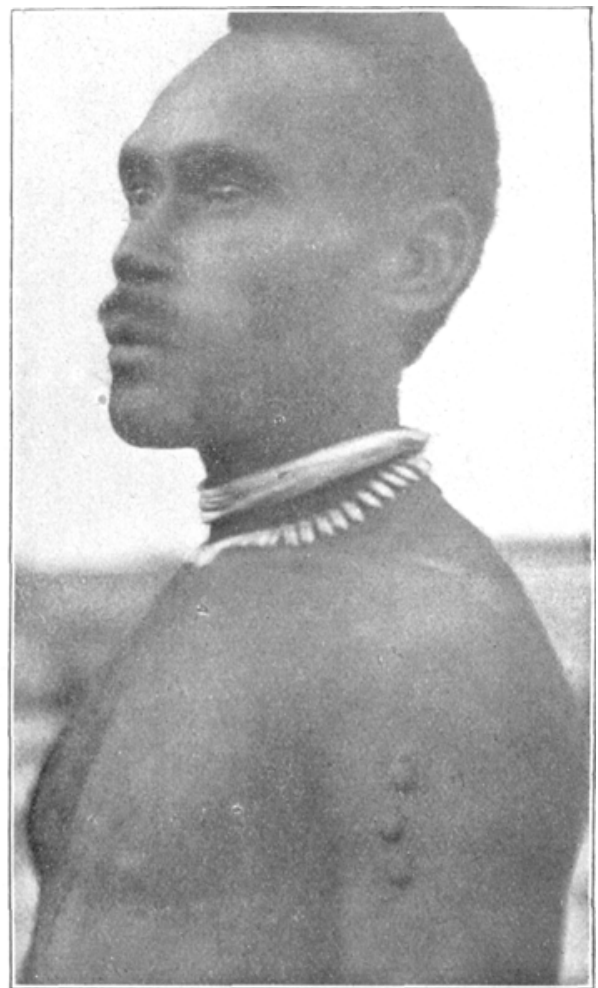

Fig. 2. Neu-Mecklenburger am 2. Tage nach der Kutanimpfung.

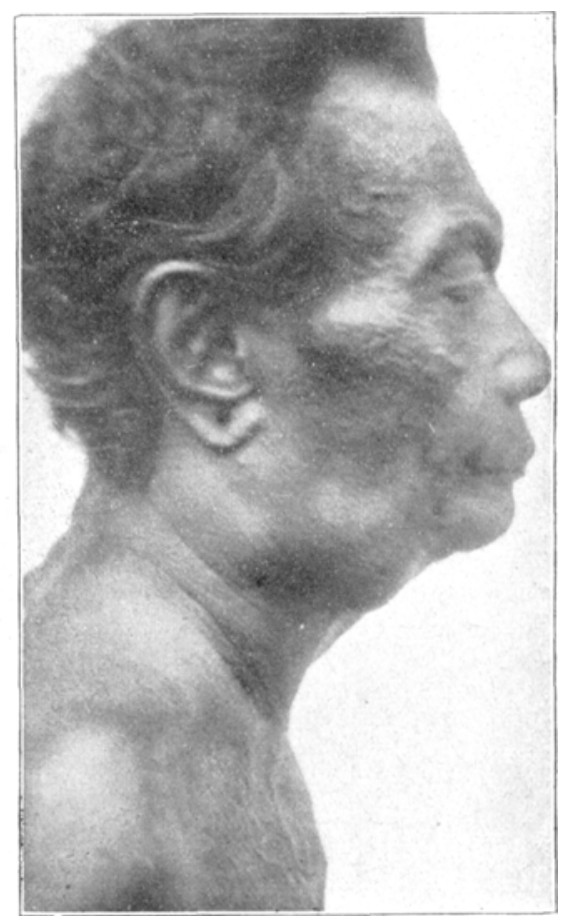

Fig. 4. Skrofuloser Greis (Karoliner).

$\mathrm{Külz}$, Die Eigenarten der Südseetuberkulose etc. 